\title{
Metabolic Stress Induces Cognitive Disturbances and Inflammation in Aged Mice: Protective Role of Resveratrol
}

\author{
Veronica Palomera-Ávalos, Christian Griñán-Ferré, Vanesa Izquierdo, \\ Antonio Camins, ${ }^{1}$ Coral Sanfeliu, and Mercè Pallàs ${ }^{1}$
}

\begin{abstract}
Inflammation and oxidative stress (OS) are key points in age progression. Both processes impact negatively in cognition and in brain functions. Resveratrol (RV) has been postulated as a potent antioxidant natural compound, with rejuvenating properties. Inducing a metabolic stress by high-fat (HF) diet in aged C56/BL6 (24 months) led to cognitive disturbances compared with control age mated and with young mice. These changes were prevented by RV. Molecular determinations demonstrated a significant increase in some inflammatory parameters ( $T N F-\alpha, C x c l 10, I L-1, I L-6$, and $C c l 3)$ in old mice, but slight changes in OS machinery. RV mainly induced the recovery of the metabolically stressed animals. The study of key markers involved in senescence and rejuvenation (mitochondrial biogenesis and Sirt1-AMPK-PGC1- $\alpha$ ) demonstrated that RV is also able to modulate the changes in these cellular metabolic pathways. Moreover, changes of epigenetic marks (methylation and acetylation) that are depending on OS were demonstrated. On the whole, results showed the importance of integrative role of different cellular mechanisms in the deleterious effects of age in cognition and the beneficial role of RV. The work presented in this study showed a wide range of processes modified in old age and by metabolic stress, weighting the importance of each one and the role of RV as a possible strategy for fighting against.
\end{abstract}

Keywords: aging, neurodegeneration, behavior, cognition, metabolic stress, mitochondrial dynamics, resveratrol

\section{Introduction}

A $\mathrm{N}$ INCREASED AGED population comprises the social landscape in the XXI century. It is estimated that between the years 2012 and 2050, the world population aged 60 and over will nearly double from 2015 to 2050, growing by $56 \%$, reaching nearly 2.1 billion. ${ }^{1}$ In addition, "oldestold" persons (people aged 80 years and over) are growing even faster than the number of old individuals. The expansion in aged population will exert an impact on the economic burden of the care and treatment of age-related diseases. Thus, a challenge in facing the aging progress is to reduce the impact of aging on health. In this respect, diet and the many bioactive substances present in food represent a novel target for interventions that may promote healthy brain aging.

Emerging evidence suggests that aside from oxidative stress (OS) and the loss of homeostatic processes with age, there are several cellular processes that may serve as a po- tential avenue for reducing the incidence of debilitating diseases in age and to halt the progression of cognitive decline associated with aging. Identifying these processes and their weight in the progression of aging is basic knowledge in considering them as possible targets to act on specific targets for ameliorating the universally harmful processes associated with aging, driving strategies toward healthy aging.

OS and inflammation are the basis of aging theories, ${ }^{2}$ and another key to cell dysfunction in aging lies in the mitochondria. Aging cells cope less efficiently with metabolism deregulations. Metabolic stress is the hypermetabolic catabolic response to severe injury or disease, and the modern notion of metabolic stress also includes disturbances in proteostasis and the activation of signaling pathways that mediate cellular stress. ${ }^{3}$ Metabolic stress has been demonstrated to induce a number of cellular process impairments such as mitochondrial changes, reduced metabolic rate, radical oxygen species (ROS) produced by mitochondria,

AU3 ${ }^{1}$ Pharmacology Section, Department of Pharmacology, Toxicology and Therapeutic Chemistry, Institute of Neuroscience, University of Barcelona, Barcelona, Spain.

${ }^{2}$ Institut d'Investigacions Biomèdiques de Barcelona (IIBB), CSIC, and IDIBAPS, Barcelona, Spain. 
and finally, the inflammation process, which can impact on the health of individuals, including the central nervous system (CNS). ${ }^{5}$ Feeding mice with a high-fat (HF) diet induced glucose metabolism disturbances with increases in weight and insulin resistance. ${ }^{6}$ In APP/PS1 mice, in an extended model of Alzheimer's disease (AD), HF produced cognitive disturbances, jointly with changes in the insulin pathway, an increase in tau kinase activity and tau hyperphosphorylation, and changes in amyloid beta production, demonstrating the role of insulin resistance in AD. ${ }^{7}$ Metabolic stress increases cognitive impairment in SAMP8, a murine model of senescence. In this model, in addition to OS and inflammation, the implication of the Wnt pathway has been related with changes in the hallmarks of $\mathrm{AD}$ characteristics in these animals. ${ }^{8}$

Diet is an important component of healthy brain aging, and the consumption of a HF diet is known to increase the risk of $\mathrm{AD}$. In addition, there is evidence that nutritional stimuli can modify epigenetic mechanisms, such as DNA methylation. ${ }^{9}$ DNA methylation/demethylation plays a crucial role in gene regulation and could also be responsible for the presentation of senescence. For example, being fed a chronic HF diet mice (from weaning to $>15$ weeks of age) altered the patterns of DNA methylation in the brain, ${ }^{10}$ and the OS and inflammation that increase by HF diet are thought to be important contributors of DNA hypermethylation. However, contradicting this is the fact that global DNA hypomethylation has been found in aging brains. ${ }^{11,12}$ Although there are other reports on AD-brain global DNA methylation changes in the opposite direction, differences are likely due to that the authors studied different brain regions and used different methylation detection techniques. ${ }^{13}$

NeuroD6 is a member of the neurogenic bHLH NeuroD family, which are proteins involved in the development and differentiation of the CNS, mediating neurogenic processes. Learning and memory processes have been related with progenitor cell proliferation and hippocampal neurogenesis. Increasing evidences support the relationship between adult hippocampal neurogenesis and learning. ${ }^{14,15}$ Moreover, it is also known that neurogenesis processes are impaired with physiological aging. ${ }^{16,17}$ It is noteworthy that recently, new role has been described for NeuroD6 as a regulator of ROS homeostasis through the triggering of an antioxidant response coordinated with the expression of key mitochondrial regulators, such as PGC- $1 \alpha$ and SIRT1, resulting in low ROS levels. ${ }^{18}$

Resveratrol is known to extend life span. ${ }^{19}$ This polyphenol has attracted wide attention because of its antioxidant and anti-inflammatory effects. ${ }^{20,21}$ Resveratrol increases AMPK activity and improves insulin sensitivity. ${ }^{22}$ AMPK is an energy sensor that regulates energy homeostasis and metabolic stress. By affecting glucose and lipid metabolism, the energy balance is closely related with obesity and type 2 diabetes. Activation of AMPK is responsible for metabolic changes through phosphorylation of downstream substrates, such as Acetyl-CoA Carboxylase and Glycogen Synthase Kinase 3 (GSK-3), which are directly related with fatty acid oxidation and glycogen synthesis, respectively. ${ }^{23}$

The goal of this work was to pinpoint the importance of metabolic stress in physiologically aged animals regarding cognition and determining the molecular changes underlying the loss of cognitive capabilities, disclaiming noncrucial processes in old animals, and focusing on new possibilities of the beneficial effect of resveratrol in the oldest.

\section{Methods}

\section{Animals}

Male C57BL/6J mice 22 months of age were randomized in four experimental groups and following 8 weeks of treatment were euthanized under anesthesia. The normal diet (ND; $n=9$ ) group had ad libitum access to a standard chow diet (2018 Teklad Global 18\% Protein Rodent Diet; Harlan Teklad, Madison, WI) and tap water; Resveratrol group (RV; $n=9)$ had access to standard chow diet enriched with the polyphenol $(1 \mathrm{~g} / \mathrm{kg}$, w/w). The HF $(n=11)$ group was fed a diet consisting of a (AIN-93G) diet modified to provide $60 \%$ of calories from fat (HF: carbohydrate:protein:fat ratio of 16:23:61\%). Finally an HF diet containing $1 \mathrm{~g} / \mathrm{kg} \mathrm{w} / \mathrm{w}$, resveratrol $(\mathrm{HF}+\mathrm{RV} ; n=9)$. Resveratrol chow (both ND and HF) was formulated to provide daily doses of $\sim 160 \mathrm{mg} / \mathrm{kg}$ to the mice. In addition, a fifth group of young ( 8 weeks) mice were added $(n=12)$ to have a young cohort. Mice were treated according to European Community Council Directive 86/609/EEC and the procedures established by the Department d'Agricultura, Ramaderia i Pesca of the Generalitat de Catalunya, Spain. Every effort was made to minimize animal suffering and to reduce the number of animals used in this study.

\section{Glucose curve}

The intraperitoneal (i.p.) glucose tolerance test was performed following 8 weeks of HF diet feeding, as described previously. ${ }^{24}$ In brief, mice were fasted overnight for 16 hours. The test was performed in a quiet room, preheated to $+30^{\circ} \mathrm{C}$. The tip of the mouse's tail was cut with the heparin-soaked (Heparina Rovi, 5000 IU/mL; Rovi S.A., Madrid, Spain) scissors 30 minutes before a $1 \mathrm{~g} / \mathrm{kg}$ i.p. glucose injection (diluted in $\mathrm{H}_{2} \mathrm{O}$ ). Blood glucose levels were measured $0,5,15,30,60$, and 120 minutes after the glucose injection with the Ascensia ELITE blood glucose meter (Bayer Diagnostics Europe, Ltd., Dublin, Ireland).

\section{Novel object recognition test}

The test was conducted in a $90^{\circ}, 2-\mathrm{arm}, 25-\mathrm{cm}$ long 20 $\mathrm{cm}$ high maze. ${ }^{25}$ Light intensity in the middle of the field was 30 lux. The objects to be discriminated were plastic figures (object A, 5.25-cm high and object B, 4.75-cm high). First, the mice were individually habituated to the apparatus for 10 minutes for 3 days. On day 4, they were submitted to a 10-minute acquisition trial (first trial) during which they were placed in the maze in the presence of two identical novel objects $(\mathrm{A}+\mathrm{A}$ or $\mathrm{B}+\mathrm{B})$ that was placed at the end of each arm. A 10-minute retention trial (second trial) occurred 2 hours later. During this second trial, objects A and B were placed in the maze, and the times that the animal took to explore the new object (tn) and the old object (to) were recorded. A discrimination index (DI) was defined as (tn-to)/ (tn+to). To avoid object preference biases, objects A and B were counterbalanced so that one half of the animals in each experimental group were first exposed to object A and then to object $\mathrm{B}$, whereas the remaining one half of the animals first saw object B, whereupon object A was presented. The 
maze, the surface, and the objects were cleaned with $70^{\circ}$ ethanol between the animals' trials to eliminate olfactory cues.

\section{Morris water maze test}

An open circular pool $(100 \mathrm{~cm}$ in diameter and $50 \mathrm{~cm}$ in height) was filled halfway with water, ${ }^{26}$ and the water temperature was maintained at $22^{\circ} \mathrm{C} \pm 1$. Two principal perpendicular axes were defined; thus, the water surface was

AU5 divided into four quadrants (NE, SE, SW, and NW), and five starting points were set (NE, E, SE, S, and SW). Four visual clues were placed on the walls of the tank (N, E, S, and W). Nontoxic white latex paint was added to make the water opaque, and a white escape platform was submerged $1 \mathrm{~cm}$ below the water level (approximately in the middle of one of the quadrants).

The animals' swimming paths were recorded by a video camera mounted above the center of the pool, and the data were analyzed with SMART $^{\circledR}$ version 3.0 statistical software. The learning phase consisted of 6 days of trials for each mouse. The animals were submitted to five trials each day starting from the positions established (in random order) and without a resting phase between each trial and the subsequent one. At each trial, the mouse was placed gently into the water, facing the wall of the pool, and allowed to swim for 60 seconds. If not able to locate the platform in the latter time period, the mouse was guided to the platform by the investigator. Animals were left on the platform each time for 30 seconds to allow spatial orientation.

The parameters measured were latency time in finding the platform, time spent in each quadrant, and distance swum for each trial; the mean was calculated for each trial day. A memory test was performed at the end of the learning days, in which the platform was removed, and the time spent by each mouse in each quadrant was measured.

\section{Open field test}

The open field test (OFT) apparatus was constructed of white plywood $(50 \times 50 \times 25 \mathrm{~cm}) .{ }^{27}$ Red lines were drawn to divide the floor into $25-\mathrm{cm}$ squares. Behavior was scored with SMART version 3.0 software, and each trial was recorded for later analysis, utilizing a camera fixed to the ceiling at a height of $2.1 \mathrm{~m}$ situated above the apparatus.
Mice were placed at the center, or at one of the four corners, of the open field and allowed to explore the apparatus for 5 minutes. After the 5-minute test, the mice were returned to their home cages, and the open field was cleaned with $70 \%$ ethyl alcohol and allowed to dry between tests. To assess the animals' habituation process to the novelty of the arena, the mice were exposed to the apparatus for 5 minutes on 2 consecutive days. The behaviors scored included Line Crossing, Center Entries, Center Stay Duration, Rearing, Defecation, and Urination. Each animal was then given a score for total locomotor activity, which was calculated as the sum of total distance, line crosses, and number of rears.

\section{Brain isolation and Western blot analysis}

Mice were euthanized 1 day after the last Morris water maze (MWM) trial was conducted and brain quickly removed from the skull. Hippocampus was dissected and frozen in powdered dry ice and maintained at $-80^{\circ} \mathrm{C}$ for further use. Tissue samples were homogenized in lysis buffer containing phosphatase and protease inhibitors (Cocktail II; Sigma), and cytosol and nuclear fractions were obtained as described elsewhere. Protein concentration was determined by the Bradford method. Twenty micrograms of protein was separated by sodium dodecyl sulfate-polyacrylamide gel electrophoresis (8\%-15\%) and transferred onto polyvinylidene difluoride membranes (Millipore). The membranes were blocked in 5\% nonfat milk in Tris-Buffered Saline solution containing $0.1 \%$ Tween 20 (TBS-T) for 1 hour at room temperature, followed by overnight incubation at $4{ }^{\circ} \mathrm{C}$ with primary antibodies diluted in TBS-T and 5\% Bovine Serum Albumin as follows: OXPHOS cocktail (1:500; MitoSciences), total GSK-3 $\beta$ (1:1000; Cell Signaling), p-Ser9 GSK-3 $\beta$ (1:1000; Cell Signaling), Dvl3 (1:1000; Cell Signaling), Dickkopf1 (1:500; Santa Cruz), $\beta$ catenin ABC (1:1000; Millipore), nuclear factor-kappa beta (NF-kB) (1:1000; Cell Signaling), Nrf1 (1:500; Santa Cruz), p-AMPK and total AMPK (1:2000; Cell Signaling), PGC$1 \alpha$ (1:500; Santa Cruz), DRP1 (1:1000; Abcam), OPA1 (1:1000; BD Transduction Laboratories $\left.{ }^{\mathrm{TM}}\right)$, Bax (1:1000; Millipore), Mitofusin 1 (1:1000; Millipore), Catalase (1:1000; Cell Signaling), and GAPDH (1:2000; Millipore). Membranes were then washed and incubated with secondary antibodies for 1 hour at room temperature. Immunoreactive proteins were

\begin{tabular}{|c|c|c|c|}
\hline AU12 & Primers & Forward sequence $\left(5^{\prime}-3^{\prime}\right)$ & Reverse sequence $\left(3^{\prime}-5^{\prime}\right)$ \\
\hline & $T N F-\alpha$ & TCGGGGTGATCGGTCCCCAA & TGGTTTGCTACGACGTGGGCT \\
\hline & Cxcl10 & GGCTAGTCCTAATTGCCCTTGG & TTGTCTCAGGACCATGGCTTG \\
\hline & $I L-1$ & ACAGAATATCAACCAACAAGTGATATTCTC & GATTCYYYCCTTTGAGGCCCA \\
\hline & $I L-6$ & ATCCAGTTGCCTTCTTGGGACTGA & TAAGCCTCCGACTTGTGAAGTGGT \\
\hline & Ccl3 & ACTGCCTGCTGCTTCTCCTACA & AGGAAAATGACACCTGGCTGG \\
\hline & iNOS & GGCAGCCTGTGAGACCTTTG & GAAGCGTTTCGGGATCTGAA \\
\hline & $\operatorname{Cox} 2$ & TGACCCCCAAGGCTCAAATA & CCCAGGTCCTCGCTTATGATC \\
\hline & Aldh2 & GCAGGCGTACACAGAAGTGA & TGAGCTTCATCCССТАCCCA \\
\hline & Neurod6 & TGCGACACTCAGCCTGAAAA & TCTGGGATTCGGGCATTACG \\
\hline & Sirt1 & AACACACACACAAAATCCAGCA & TGCAACCTGCTCCAAGGTAT \\
\hline & Dnmt $3 b$ & TGCCAGACCTTGGAAACCTC & GCTGGCACCCTCTTCTTCAT \\
\hline & Hdacl & TCACCGAATCCGCATGACTC & TCTGGGCGAATAGAACGCAG \\
\hline & Hdac2 & CTATCCCGCTCTGTGCCCT & GAGGCTTCATGGGATGACCC \\
\hline & $\beta$-Actin & CAACGAGCGGTTCCGAT & GCCACAGGTTCCATACCCA \\
\hline
\end{tabular}


visualized utilizing an Enhanced ChemiLuminescencebased Detection Kit (ECL Kit; Millipore), and digital images were acquired using a ChemiDoc XRS+System (Bio-Rad). Band intensities were quantified by densitometric analysis using Image Lab software (Bio-Rad), and values were normalized to GAPDH.

\section{Global DNA methylation quantification}

For global DNA, methylation was performed according to the manufacturer's instructions, first using the FitAmpTM Blood and Cultured Cell DNA Extraction Kit, which is designed for rapid isolation of pure genomic DNA from a small amount of blood or mammalian cells. The second part was carried out using the MethylFlash Methylated DNA Quantification Kit (Epigentek, Farmingdale, NY). Briefly, methylated DNA was detected utilizing capture and detection antibody to $5-\mathrm{mC}$ and then quantified colorimetrically by reading absorbance at $450 \mathrm{~nm}$ using the Microplate Photometer. The absolute amount of methylated DNA (proportional to the optical density [OD] intensity) was measured and was quantified using a standard curve plotting OD values versus five serial dilutions of control methylated DNA $(0.5-10 \mathrm{ng})$.

\section{RNA extraction and gene expression determination}

Total RNA isolation was carried out by means of Trizol reagent following the manufacturer's instructions. RNA content in the samples was measured at $260 \mathrm{~nm}$, and sample purity was determined by the A260/280 ratio in a NanoDrop $^{\mathrm{TM}}$ ND-1000 (Thermo Scientific). Samples were also tested in an Agilent 2100B Bioanalyzer (Agilent Technologies) to determine the RNA integrity number. Reverse

AU6 transcription-polymerase chain reaction (RT-PCR) was performed as follows: $2 \mu \mathrm{g}$ of messenger RNA (mRNA) was reverse-transcribed using the High Capacity (complementary DNA) cDNA Reverse Transcription Kit (Applied Biosystems). Real-time quantitative PCR (qPCR) was utilized to quantify the mRNA expression of inflammatory genes interleukin 6 and 1 ( $I L-6$ and $I L-1)$, tumor necrosis factor alpha $(T N F-\alpha), \mathrm{C}-\mathrm{X}-\mathrm{C}$ motif chemokine $(\mathrm{Cxcl10}), \mathrm{C}-$ $\mathrm{C}$ motif ligand 3 ( $\mathrm{Ccl} 3$ ), Aldehyde dehydrogenase 2 (Aldh2), inducible Nitric Oxide Synthase (iNOS), Cyclooxygenase 2 (Cox2), and aldehyde oxidase 1 (Aoxl) and epigenetic enzyme genes DNA (cytosine-5-)-methyltransferase 3 alpha (Dnmt3a), DNA (cytosine-5-)-methyltransferase 3 beta (Dnmt $3 b)$, Histone deacetylase 1 (Hdacl), Histone deacetylase 2 (Hdac2), Sirtuin 1 (Sirt1), and Neuronal differentiation 6 (Neurod6). Normalization of expression levels was performed with actin for SYBR Green and TATA-binding protein $(T b p)$ for TaqMan. time PCR was performed on the StepOnePlus Detection

Table 2. QRT-PCR Primer SEQuences UsEd WITH TAQMaN PRobe

\begin{tabular}{lc}
\hline Primers & Probes \\
\hline Dnmt3a & Mm00432881_m1 \\
Tbp & Mm00446971_m1 \\
\hline
\end{tabular}

System (Applied Biosystems) using the SYBR Green PCR Master Mix (Applied Biosystems). Each reaction mixture contained $7.5 \mu \mathrm{L}$ of cDNA, whose concentration was $2 \mu \mathrm{g}$, $0.75 \mu \mathrm{L}$ of each primer (whose concentration was $100 \mathrm{nM}$ ), and $7.5 \mu \mathrm{L}$ of SYBR Green PCR Master Mix (2X).

Data were analyzed utilizing the comparative Cycle threshold method $(\Delta \Delta \mathrm{Ct})$, where the actin transcript level was used to normalize differences in sample loading and preparation. Each sample $(n=4-5)$ was analyzed in
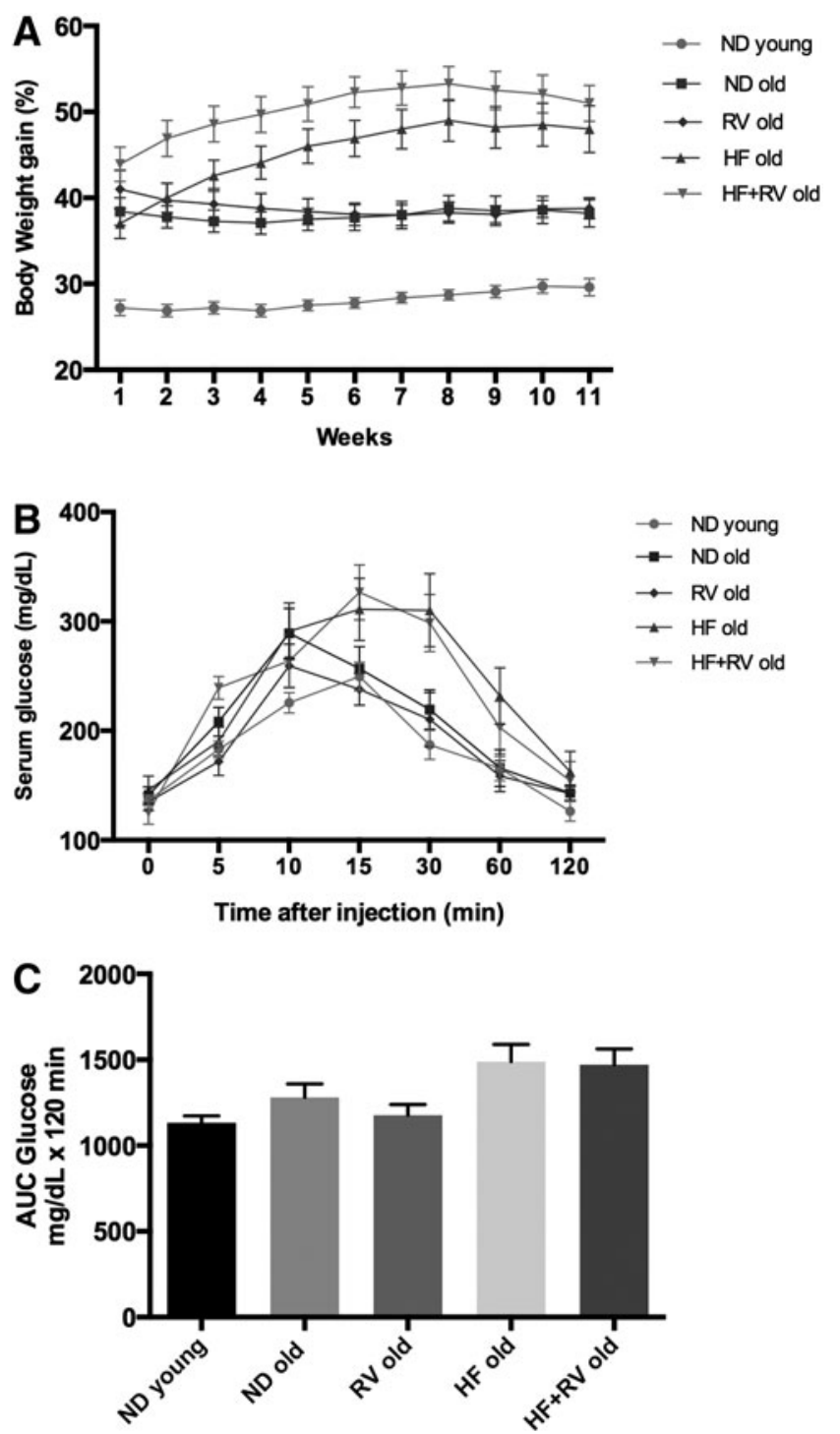

FIG. 1. Results of body weight, caloric intake, and glucose test in C57BL/6J mice. (A) Body weights of mice fed with normal diet (ND young $n=12$ and ND old $n=9$ ), resveratrol (RV old $n=9$ ), high-fat diet (HF old $n=11$ ), or high-fat diet plus resveratrol (HF+RV old $n=9)$. (B) Plasma levels of glucose $1 \mathrm{~g} / \mathrm{kg}$ i.p. administration. (C) Glucose AUC after 2 hours glucose injection. Data represented observed mean \pm SEM. For compared groups, we did ANOVA One way. *Significant differences between ND old groups versus ND young and RV groups, $p<0.05$. ${ }^{\$}$ Significant differences between ND old groups versus $\mathrm{HF}$ and $\mathrm{HF}+\mathrm{RV}$ groups, $p<0.05$. ANOVA, analysis of variance; AUC, area under the curve; HF, high fat; i.p., intraperitoneal; ND, normal diet; RV, resveratrol; SEM, standard error of the mean. 
triplicate, and results represented the $n$-fold difference of transcript levels among different samples.

\section{Statistical analysis}

Data are expressed as mean \pm standard error of the mean. The trapezoidal rule was used to determine area under the curve. Means were compared with two-way analysis of variance and post hoc analysis. Comparisons were performed for young/ND old/RV old and ND old/HF old/ $\mathrm{HF}+\mathrm{RV}$ old. Statistical significance was considered when $p$-values were $<0.05$. Statistical outliers were performed out with the Grubbs test and were removed from analysis.

\section{Results}

\section{Weight evolution and glucose tolerance test}

Mouse groups under HF diet exhibited exponential increase in body weight (BW) for up to 8 weeks (Fig. 1A). This gain was absent in ND old animals and was slightly present in young animals (Fig. 1A). Persistent high-blood glucose levels between 20 and 90 minutes of glucose tolerance test at 8 weeks post HF diet treatment in mice indicated systemic failure to reduce blood glucose, implying sustained insulin resistance induced by HF diet during 8 weeks (Fig. 1B). When RV was added to diet (ND and HF), no changes were observed in BW.

\section{Cognitive studies: learning and memory}

Results obtained in the OFT indicated that the HF groups exhibited a significant increase in time spent in the center zone with respect to ND group old (Fig. 2A), paralleling the $4 \mathrm{~F} 2$ time spent in border zone (Fig. 2B); RV increased, in a nosignificant manner, the time spent in center open field compared with ND old (Fig. 2A); and no differences were observed in locomotor activity (Fig. 2C). In the novel object recognition test (NORT), we found that mice fed with $\mathrm{HF}$ diet had a lower DI with respect to ND old (Fig. 2D), demonstrating that SAMP8 fed with HF diet exhibited impaired memory capabilities compared with old SAMP8 fed with ND or HF-RV.

The results obtained in spatial learning acquisition and retention in the MWM test are illustrated in Figure 3A and Table 3. All mouse groups were able to learn along the trial days, although young animals accomplished this faster than old animals. Results obtained in MWM memory test were very variable across the different parameters studied, probably because the advanced age in addition to the weight gain in HF animals reduced the reliability of this test under our experimental condition. Therefore, despite a trend to lower
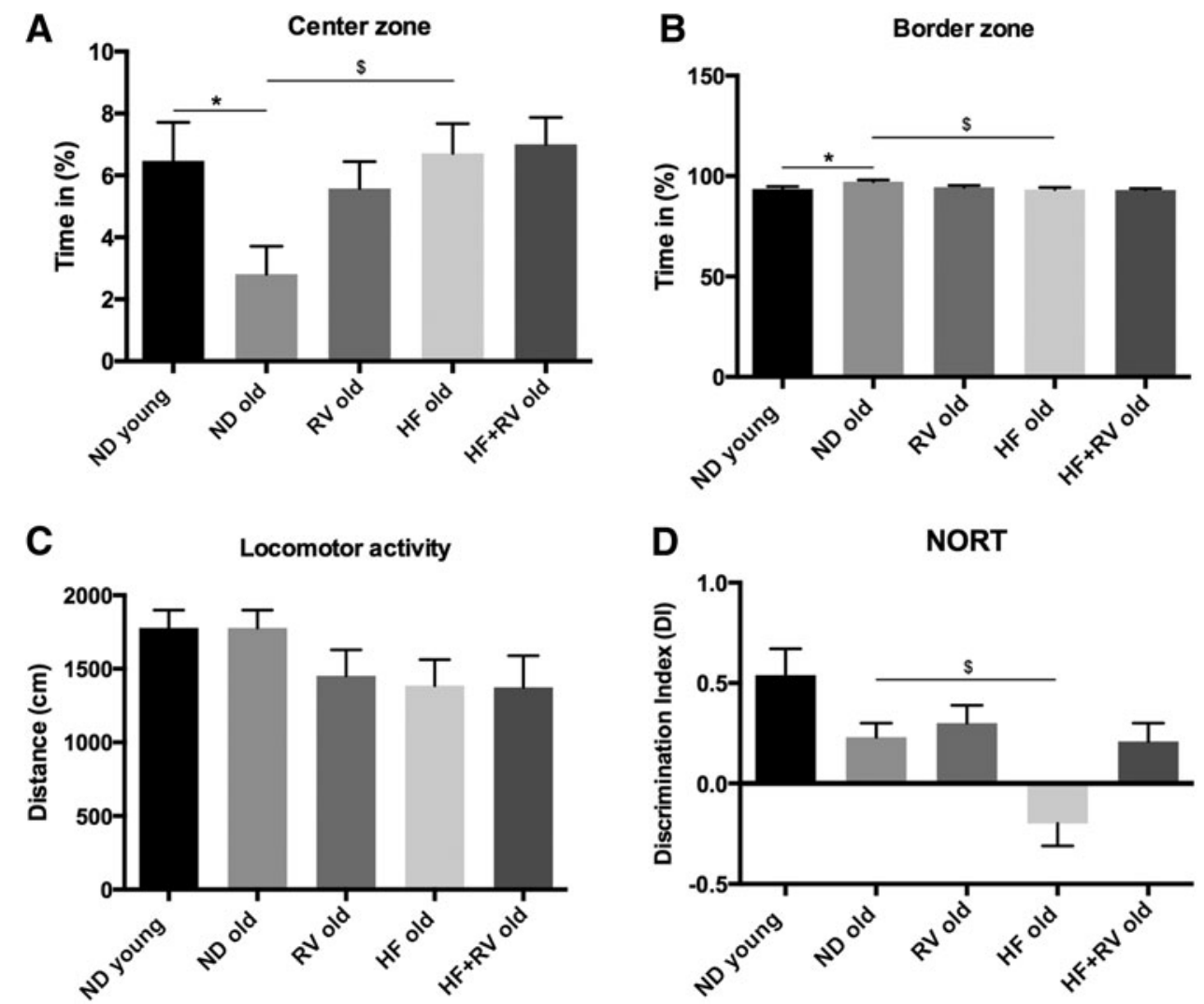

FIG. 2. Results of OFT and the NORT in C57BL/6J young and old fed with normal diet (ND young $n=12$ and ND old $n=9$ ), resveratrol (RV old $n=9$ ), high-fat diet (HF old $n=11$ ), or high-fat diet plus resveratrol (HF+RV old $n=9$ ). (A) Time spent in center zone in the OFT. (B) Time spent in border zone in the OFT. (C) Locomotor activity in the OFT. (D) Discrimination index in the NORT. Data represented observed mean \pm SEM. For compared groups, we did ANOVA One way. *Significant differences between ND old groups versus ND young and RV groups, $p<0.05$. \$Significant differences between ND old groups versus HF and HF+RV groups, $p<0.05$. NORT, novel object recognition test; OFT, open field test. 

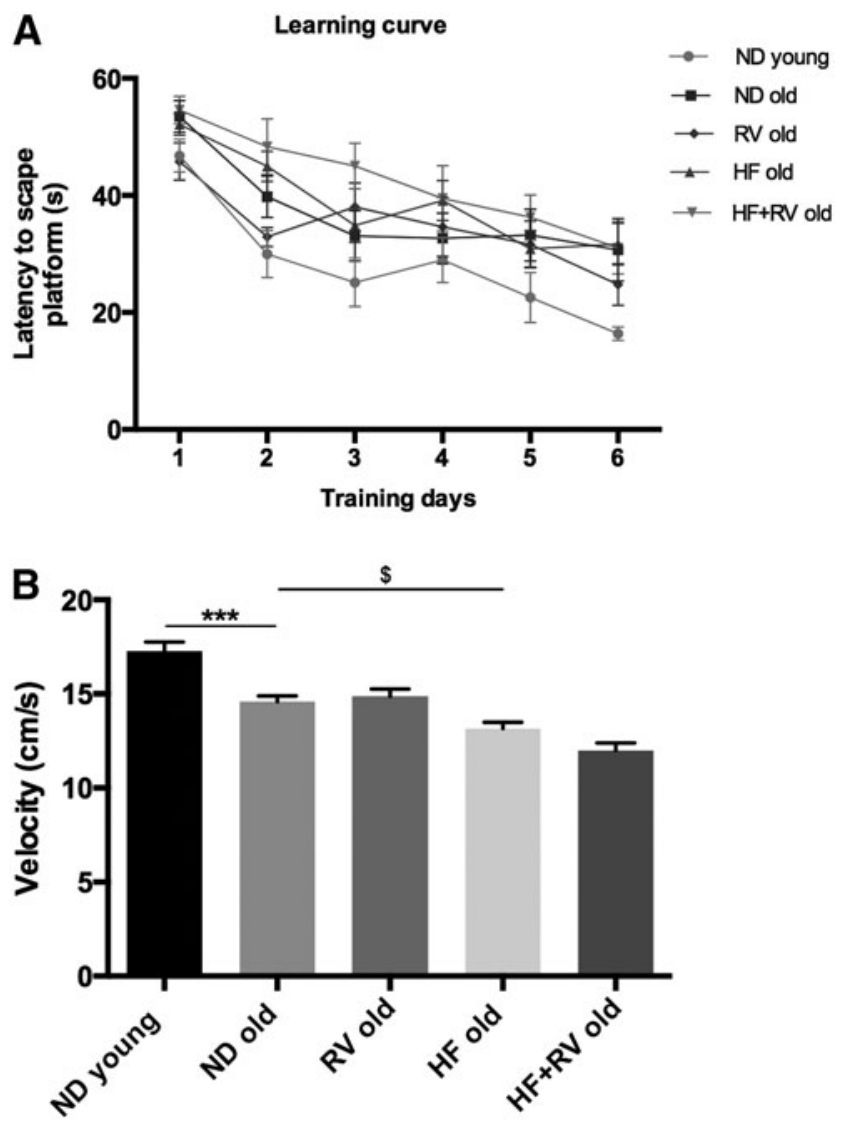

FIG. 3. Results of spatial learning and memory in the Morris water maze in C57BL/6J young and old fed with normal diet (ND young $n=12$ and ND old $n=9$ ), resveratrol (RV old $n=9$ ), high-fat diet (HF old $n=11$ ), or high-fat diet plus resveratrol $(\mathrm{HF}+\mathrm{RV}$ old $n=9)$. (A) Escape latency time to reach the hidden platform during training days. (B) Velocity. Data represented observed mean \pm SEM. For compared groups we did ANOVA One way. *Significant differences between ND old groups versus ND young and $\mathrm{RV}$ groups, $p<0.05, * * p<0.01, * * * p<0.001$. ${ }^{\$}$ Significant differences between ND old groups versus $\mathrm{HF}$ and $\mathrm{HF}+\mathrm{RV}$ groups, $p<0.05$.

performance by HF old, no significant memory differences were obtained (from $20 \%$ to $24 \%-27 \%$ time in platform; Table 3). The most robust result was swimming velocity (Fig. 3B). This parameter revealed a significant decrease in swim velocity in old animals with respect to young mice and also demonstrated global amelioration in RV-fed animals.

\section{Molecular and biochemical changes induced} by HF in old mice: effects of resveratrol

$\mathrm{HF}$ induces a significant increase in the gene expression of inflammatory cytokines $T N F-\alpha, C x c l 10, I L-1, I L-6$, and $\mathrm{Ccl} 3$ in old mice in reference to ND. RV induced significant reduction in the expression of these cytokines in HF-fed animals, but no change was determined in ND-fed old mice after RV administration (Fig. 4A-E). Thus, the preventive $4 \mathrm{~F} 4$ effect of RV appears only under metabolic stress induced by $\mathrm{HF}$, but not under ND conditions. It is noteworthy that only $T N F-\alpha$ and $C c l 3$ were increased in ND old animals, whereas the remaining cytokines determined did not change in old compared with young animals.

We found statistical differences in $i N O S$ gene expression between ND and HF (Fig. 5A). However, RV administration 4 F5 did not modify iNOS gene expression in any experimental groups, although a nonsignificant decrease can be observed in HF-RV animals (Fig. 5A). Aoxl (data not shown), Cox2, or Aldh2 gene expression did not demonstrate significant changes in young, ND, or HF mice. RV was able to increase in Aldh2 ND old mice in a significant manner (Fig. 5B). NF$\mathrm{kB}$ is a protein complex that is involved in cellular responses to OS in cytokines, among others. Subsequently, gene expression as an OS effector was studied. We did not find statistical differences between ND and HF, but RV was able to reduce the active subunit of this transcriptional factor significantly in ND-RV, and there was a nonsignificant trend to diminish this in HF-RV in comparison with HF (Fig. 5C). In contrast, catalase (CAT) levels were studied; we found statistical differences between ND and HF, and a marginal reversion was observed in RV-fed animals (Fig. 5D). These results reinforced those obtained with $i N O S$, indicating weak changes in the level of OS induced by HF in old compared with young animals. Finally, cytoplasmic and nuclear protein levels for nuclear factor erythroid 2-related factor 1 (Nrf1) were determined, but no significant changes were observed (Fig. 5E-F).

To determine whether HF affects mitochondrial respiratory chain protein elements, OXPHOS was performed by Western blot (Fig. 6A). Analysis revealed that no significant $<$ F6 changes in OXPHOS components took place in the HF group; however, a significant increase in CIII-UQCRC2, CIV-MTCO1, and CII-SDHB complexes was found in RV-treated animals (Fig. 6C-E). Accordingly, with slight

Table 3. Parameters Measured in the Morris Water Maze in C57Bl/6J Young and Old Fed with Normal Diet (ND Young $N=12$ and ND Old $N=9$ ), Resveratrol (RV Old $N=9$ ), High-Fat Diet (HF Old $N=11$ ), or High-Fat Diet Plus Resveratrol (HF+RV Old $N=9$ )

\begin{tabular}{lrrrrr}
\hline & ND young & ND old & HF old & HF+RV old & RV old \\
\hline Latency to platform (first day) & $43.81 \pm 1.11$ & $47.53 \pm 1.37$ & $48.17 \pm 0.94$ & $46.67 \pm 1.67$ & $42.46 \pm 1.59$ \\
Latency to platform (last day) & $23.17 \pm 1.00$ & $28.07 \pm 1.30$ & $30.71 \pm 0.64$ & $29.03 \pm 0.98$ & $29.60 \pm 0.85$ \\
Distance in zone platform (\%) & $27 \pm 1.48$ & $27 \pm 1.65$ & $21 \pm 1.44$ & $24 \pm 3.30$ & $26 \pm 3.15$ \\
Time in zone platform (\%) & $26 \pm 2.16$ & $25 \pm 2.74$ & $20 \pm 1.59$ & $25 \pm 4.05$ & $25 \pm 3.22$ \\
Entries in zone platform & $5 \pm 0.67$ & $4 \pm 0.68$ & $3 \pm 0.48$ & $4 \pm 0.5$ & $3 \pm 0.31$ \\
Velocity (cm/s) & $17.27 \pm 0.48$ & $14.59 \pm 0.29$ & $13.16 \pm 0.32$ & $11.99 \pm 0.40$ & $14.85 \pm 0.41$ \\
\hline
\end{tabular}

Results are expressed as a mean \pm standard error of the mean.

$\mathrm{HF}$, high fat; ND, normal diet; RV, resveratrol. 

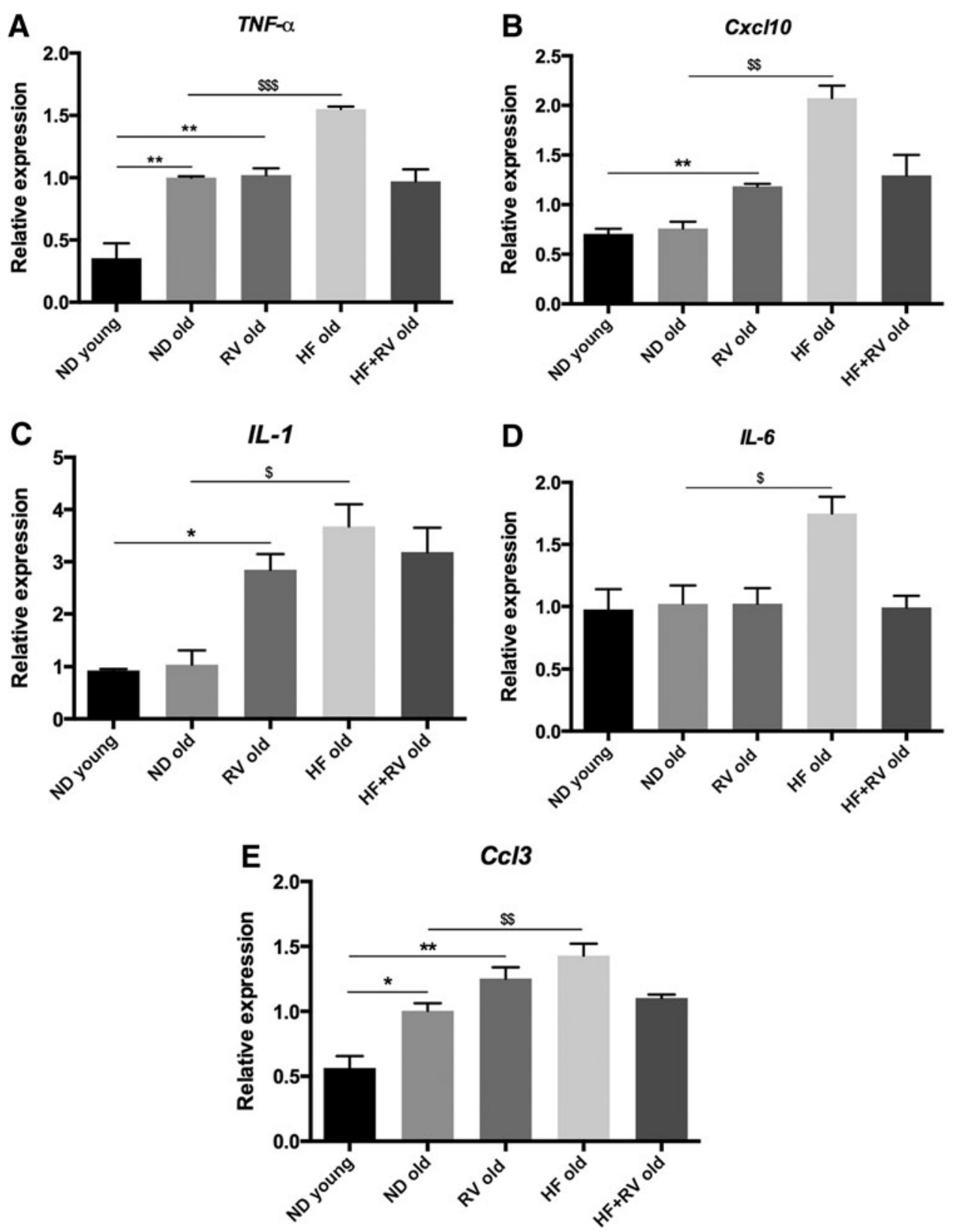

FIG. 4. Pro-inflammatory gene expression for (A) $T N F-\alpha$; (B) $C x c l 10 ;$ (C) $I L-1$; (D) $I L-6$; and (E) $C c l 3$ in C57BL/6J young and old fed with normal diet (ND young $n=12$ and ND old $n=9$ ), resveratrol (RV old $n=9$ ), high-fat diet (HF old $n=11)$, or high-fat diet plus resveratrol ( $\mathrm{HF}+\mathrm{RV}$ old $n=9)$. Gene expression levels were determined by real-time PCR. Mean \pm SEM performed in triplicate is represented. For compared groups, we did ANOVA One way. *Significant differences between ND old groups versus ND young and RV groups, $p<0.05$, $* * p<0.01$. ${ }^{\$}$ Significant differences between ND old groups versus $\mathrm{HF}$ and $\mathrm{HF}+\mathrm{RV}$ groups, $p<0.05,{ }^{\$ \$} p<0.01,{ }^{\$ \$} p<0.001$.

changes in mitochondrial function, no changes were determined in fission or fusion processes through OPA1, DRP1, and Mitofusin 1 proteins (Fig. 6G-I).

NeuroD6 expression demonstrated a diminution in old animals, and $\mathrm{RV}$ recovered the expression of this enzyme to

F7 young mice levels (Fig. 7A). In addition, Sirt1 gene expression levels presented significant increase in ND-RV in relation to ND, but this profile did not occur under HF diet (Fig. 7B). In contrast, PGC- $1 \alpha$ increased in HF with reference to ND, while RV dietary supplementation recovered PGC- $1 \alpha$ protein levels in ND, but not in HF-fed animals (Fig. 7C).
In reference to AMPK signaling, we found an increase in pAMPK levels in old animals. The increase in phosphorylation levels was higher in HF group in reference to ND. RV administration decreased pAMPK levels, achieving a profile similar to that of ND mice (Fig. 7D). Moreover, the proapoptotic protein Bax was analyzed; no changes were observed in protein levels by RV treatment (Fig. 7E),

Wnt pathway can be modulated by metabolic stress. GSK-3 $\beta$ is one of the effectors of this pathway. RV was able to increase the inactive form of GSK-3 $\beta$ (pSer9) protein levels, which reached significance in HF-fed animals 


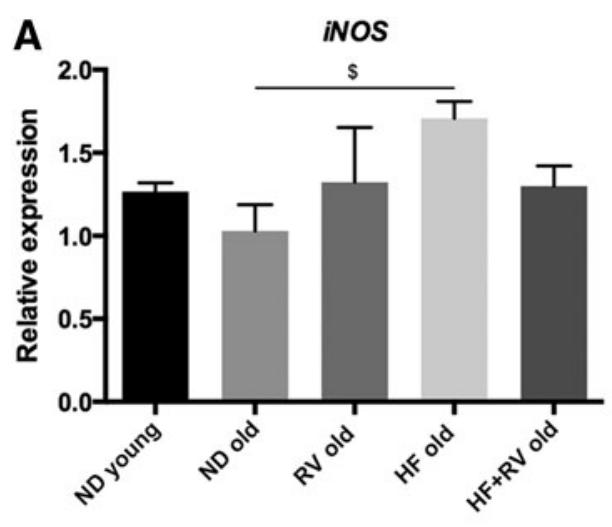

C

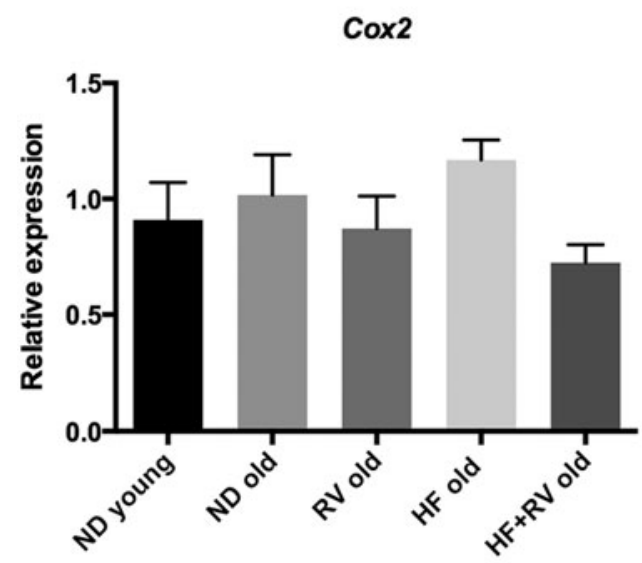

E

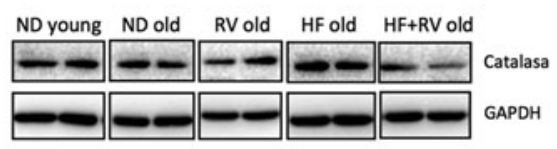

Catalasa

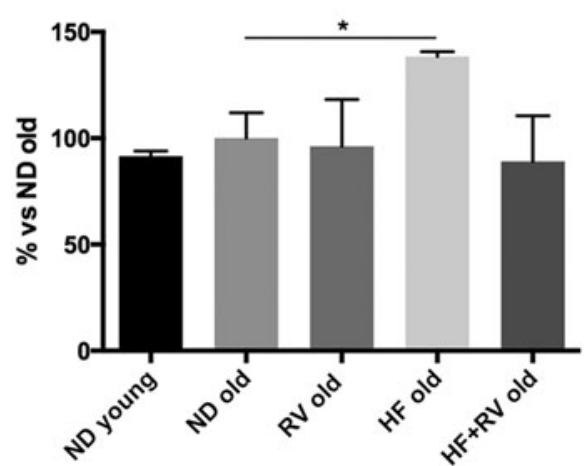

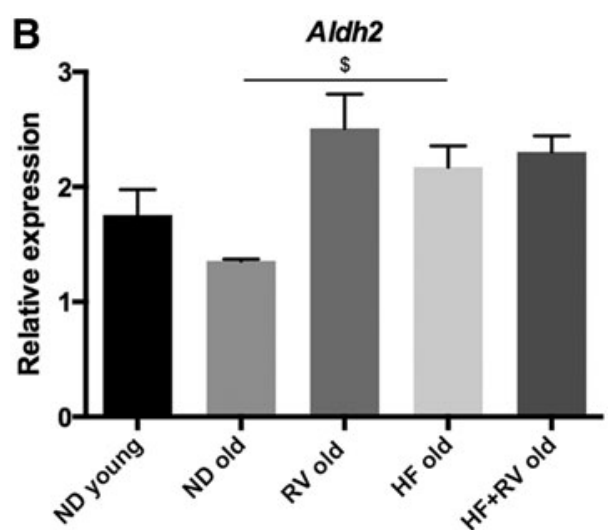

D ND young ND old RV old $\mathrm{HF}$ old $\mathrm{HF}+\mathrm{RV}$ old

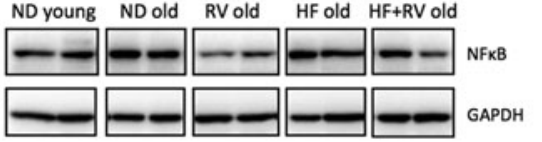

NFkB

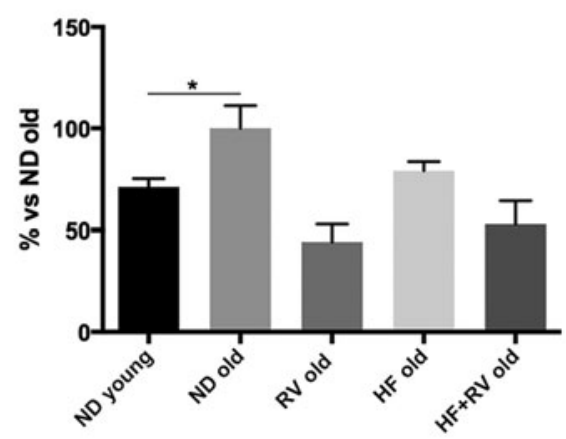

$\mathbf{F}$

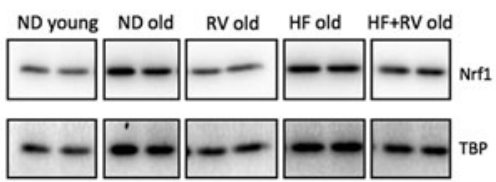

Nrf1 nucleus

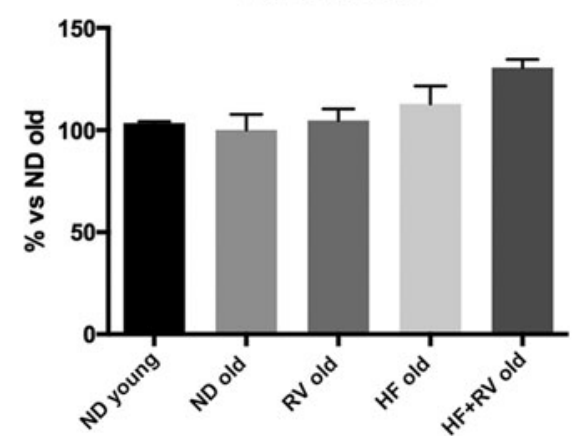

FIG. 5. Oxidative stress markers in young and old mice C57BL/6J. Gene expression levels were determined by real-time PCR. (A) iNOS, (B) Aldh2, (C) Cox2, representative Western blot and quantification for (D) NFkB, (E) Catalase, (F) Nrf1 nucleus, and (G) Nrf1 cytoplasm. Mean \pm SEM performed in triplicate is represented in PCR; for Western blot the values are adjusted to $100 \%$ for levels C57BL/6J old with Normal Diet. For compared groups, we did ANOVA One way. *Significant differences between ND old groups versus ND young and RV groups, $p<0.05$. Significant differences between ND old groups versus $\mathrm{HF}$ and $\mathrm{HF}+\mathrm{RV}$ groups, $p<0.05$. 
A

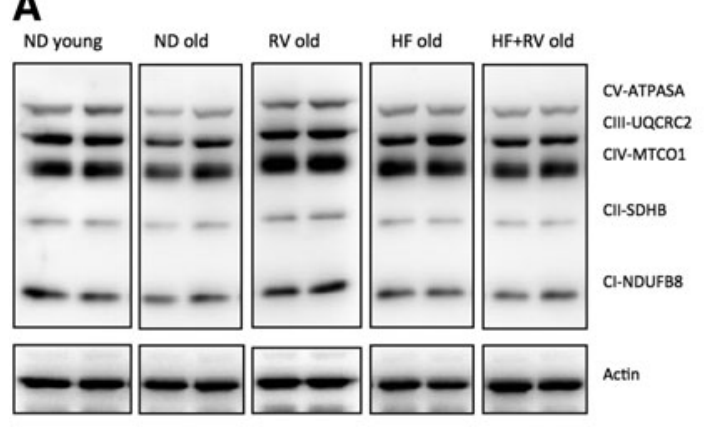

C

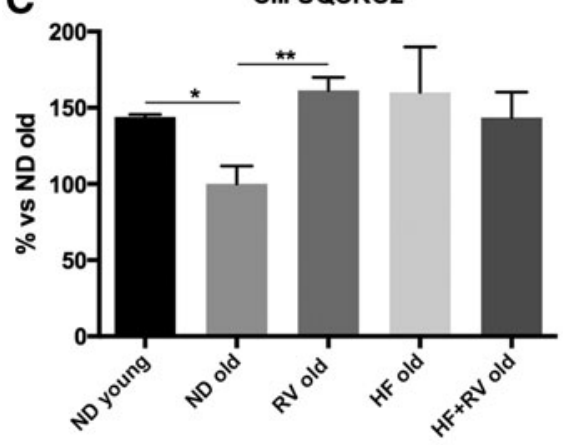

E

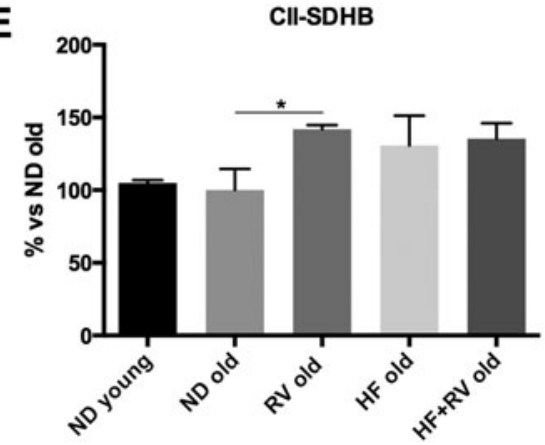

B

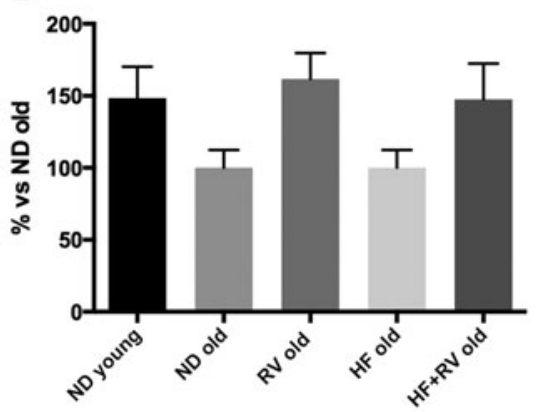

D

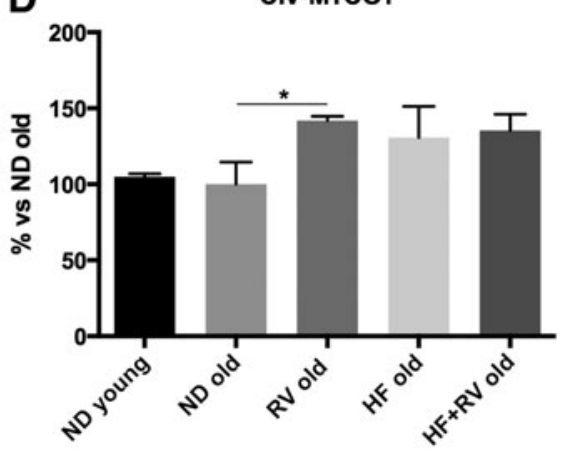

$\mathbf{F}$

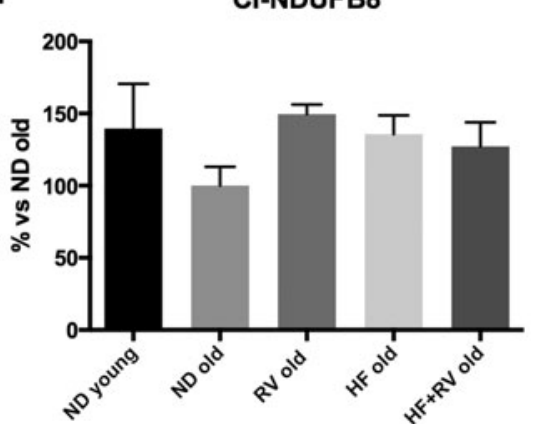

FIG. 6. (A) Representative Western blot OXPHOS and quantifications for (B) CV-ATPASA, (C) CIII-UQCRC2, (D) CIV-MTCO1, (E) CII-SDHB, (F) CI-NDUFB8, (G) OPA1, (H) Mitofusin1, and (I) DRP1 in C57BL/6J young and old fed with normal diet (ND young $n=12$ and ND old $n=9$ ), resveratrol (RV old $n=9$ ), high-fat diet (HF old $n=11$ ), or high-fat diet plus resveratrol $(\mathrm{HF}+\mathrm{RV}$ old $n=9)$. Bars represent mean $\pm \mathrm{SEM}$, and values are adjusted to $100 \%$ for levels $\mathrm{C} 57 \mathrm{BL} / 6 \mathrm{~J}$ old with Normal Diet. For compared groups, we did ANOVA One way. *Significant differences between ND old groups

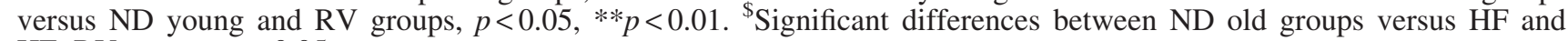
$\mathrm{HF}+\mathrm{RV}$ groups, $p<0.05$.

F8 (Fig. 8A). $\beta$-catenin, a target of this kinase through Wnt pathway, was activated in RV-fed animals (both ND and HF diet) (Fig. 8B). In contrast, no changes were observed in DKK or Dvl3 protein levels in the experimental groups of interest (Fig. 8C, D).

Aging and HF were described to induce changes in epigenetic hallmarks. The levels of DNA methylation, DNA MethyTtransferases (DNMT), histone demethylases, and Histone DeACetylases (HDAC), were determined by RT-

F9 PCR in all groups (Fig. 9A-E). Global methylation was studied measuring $5 \mathrm{mC}$ levels; we observed that RV reduced $5 \mathrm{mC}$ levels in ND mice (Fig. 9A). DNMT did not exhibit changes with age or diet (Fig. 9B, C). Only a reduction in Dnmt3b in the RV group with respect to the ND group was shown in Figure 9C. In Hdacl expression levels, there were no significant results (Fig. 9D). Conversely, Hdac2 showed an increase in gene expression in old ND animals in reference to young mice, and RV was able to revert gene expression to young mouse levels (Fig. 9E).

\section{Discussion}

Reductions in specific cognitive abilities, such as mental speed, executive function, and episodic memory, are commonly experienced in aging. ${ }^{28}$ The excessive production of reactive oxidative species (ROS) is associated with inflammation, ${ }^{29}$ and aged rodents exhibited exaggerated neuroinflammation and memory deficits in response to OS. ${ }^{30} \mathrm{We}$ found a clear inflammatory landscape in hippocampus in old in comparison with young mice, which could be the 

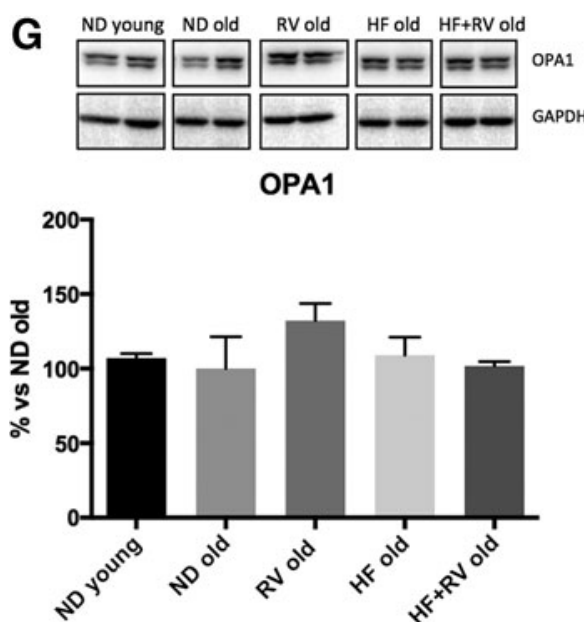
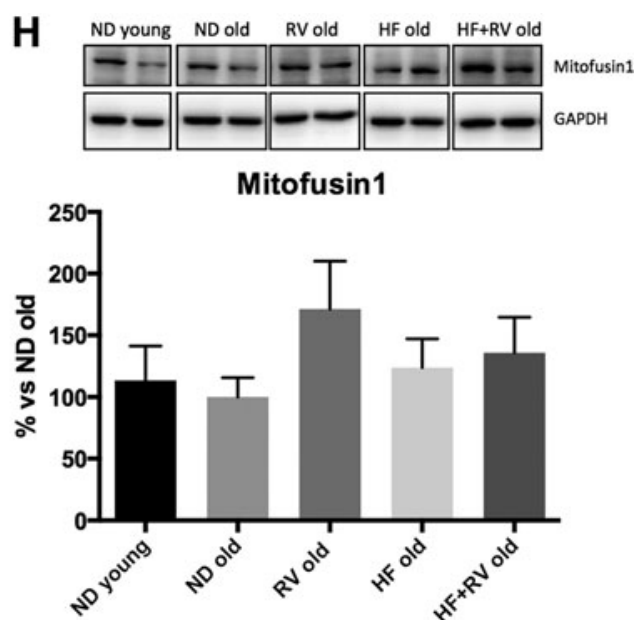
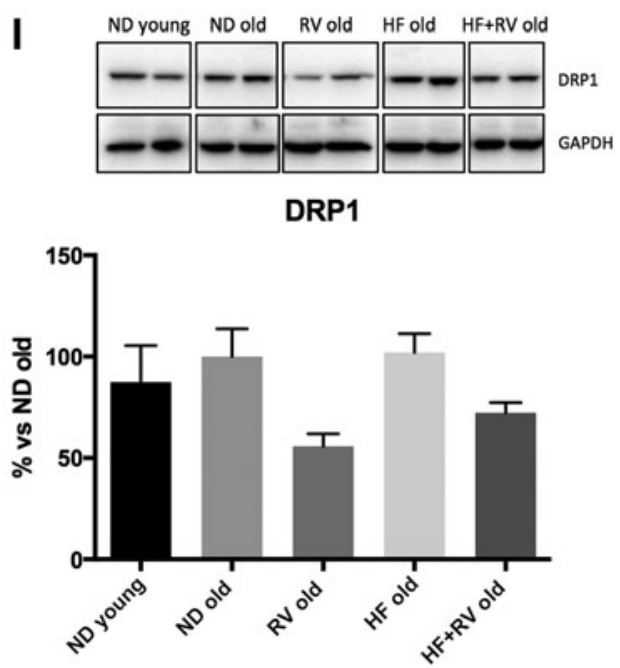

FIG. 6. (Continued)

consequence of the OS condition in aged animals, finally rendering cognitive impairment. Furthermore, old mice showed a decrease in Neurod6 and PGC-1alpha, which are described as physiological energetic sensors.

In reference to behavior parameters, in the NORT test, a drop was demonstrated in DI values obtained in HF-fed mice after NORT in comparison with young and old animals. RV was able to prevent cognition impairment in HFfed mice. MWM results reinforced the effect of RV, but did not evidence significant changes among untreated animals, probably due to the physical condition of aged animals. OFT evaluates motor activity, but also assesses disinhibition behavior based on the aversion of mice to open spaces. HF induced disinhibition behavior in reference to ND-fed mice that was reversed by RV. Dementia can be gated to this disinhibition, and AD models have shown increased disinhibition. ${ }^{31,32}$ Thus metabolic stress induced by HF led to behavior and cognitive disturbances in C57BL/6J old mice that were prevented by RV.

However, enzymes related with OS (iNOS, CAT, COX2) did not exhibit significant differences between young and old animals, although in a number of parameters measured, distinct tendencies can be observed, indicating that at older ages, a standby point in oxidative markers appears to be achieved in this strain. Accordingly, no significant changes were found in Nrf1 described as a transcription factor that controls the basal and induced expression, regulating the physiological and pathophysiological outcomes of oxidant exposure.

Because we studied aged animals, based on an impaired condition, it should be feasible to hypothesize that aging reduced the possibility that HF delivered a high degree of metabolic-stress impairment, consequently high OS, as reported by several authors in different animal models at younger ages. ${ }^{7,8,23}$

Inducing metabolic stress in the 24-month-old C57BL/6J strategy exhibited significant changes in several oxidative markers, including iNOS and CAT, but none were found in Aox1, Aldh2, Cox2, or Nrf1. RV did not induce effective changes in oxidant parameters, except for Aldh2, an enzyme implicated in detoxifying aldehydes that accumulate through metabolism. ${ }^{33,34}$ It is suggested that Aldh2 dysfunction may contribute to a variety of human diseases, including cardiovascular diseases, diabetes, neurodegenerative diseases, stroke, and cancer. The high degree of dispersion did not allow us to determine changes in $i N O S$ or CAT expression in $\mathrm{RV}$-fed mice.

Despite the weak OS process present in our model, proinflammatory activity, a process tightly bound to OS, ${ }^{23}$ was 
A

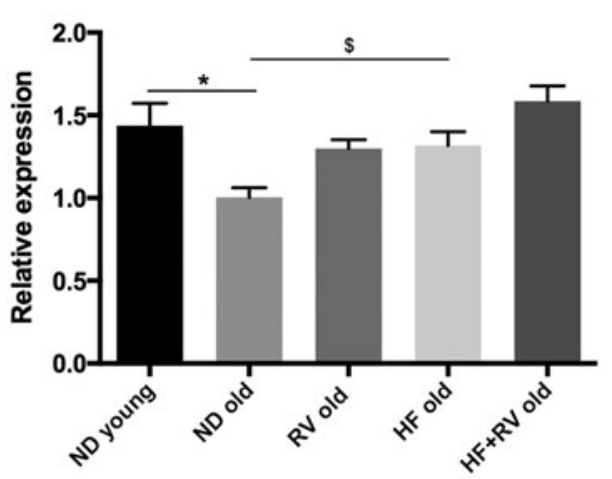

C

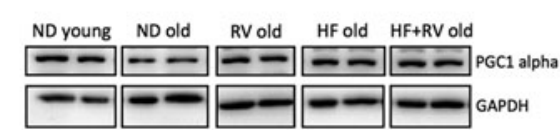

PGC1 alpha

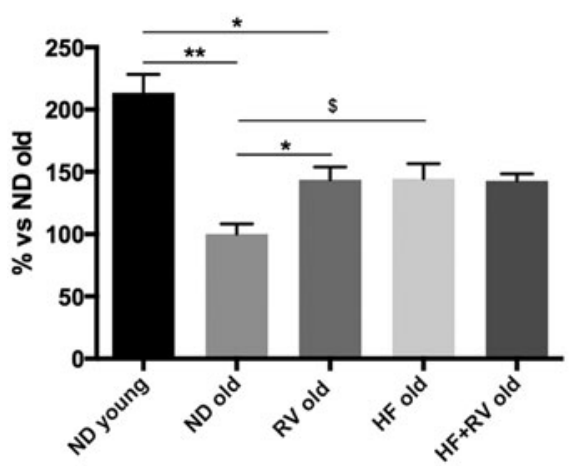

B

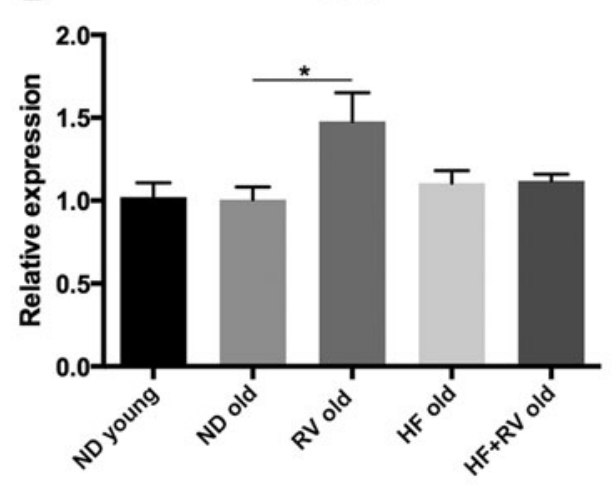

D

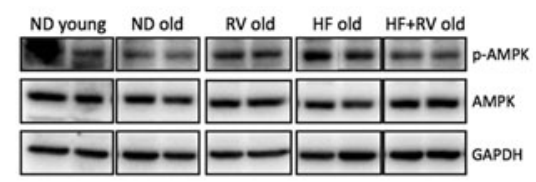

Ratio PAMPKIAMPK total

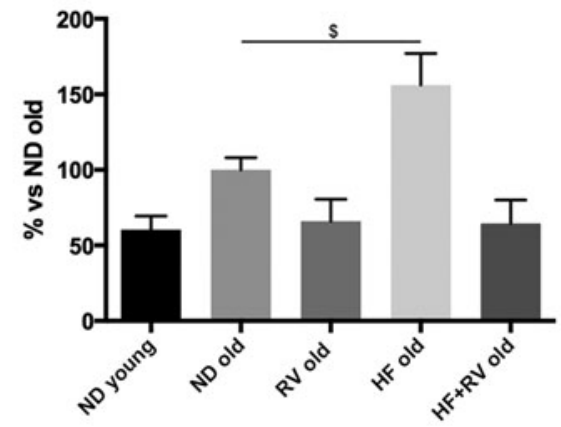

E
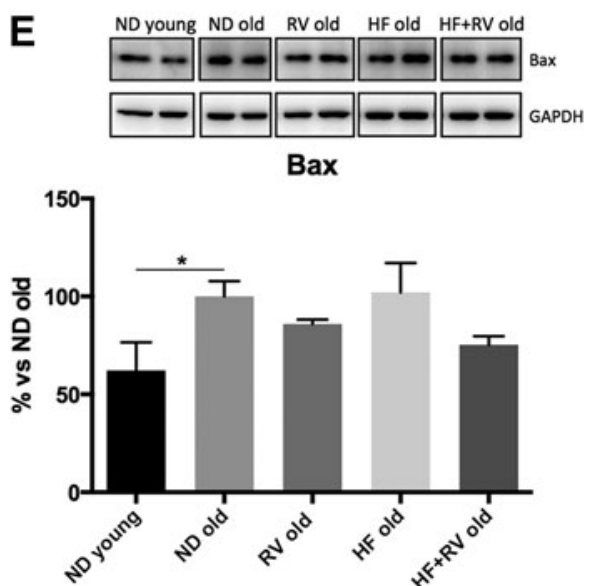

FIG. 7. Gene expression for (A) Neurod6 and (B) Sirt1 and representative Western blot and quantifications for (C) PGC-1 alpha, (D) Ratio pAMPK/AMPK total, (E) Bax in C57BL/6J young and old fed with normal diet (ND young $n=12$ and ND old $n=9$ ), resveratrol (RV old $n=9$ ), high-fat diet (HF old $n=11)$, or high-fat diet plus resveratrol $(\mathrm{HF}+\mathrm{RV}$ old $n=9$ ). Mean \pm SEM performed in triplicate is represented in PCR; for Western blot the values are adjusted to $100 \%$ for levels C57BL/6J old with ND. For compared groups, we did ANOVA One way. *Significant differences between ND old groups versus ND young and RV groups, $p<0.05, * * p<0.01$. ${ }^{\$}$ Significant differences between ND old groups versus HF and $\mathrm{HF}+\mathrm{RV}$ groups, $p<0.05$.

demonstrated in aged animals. Inflammation comprises a defensive response to a number of insults, including an oxidative environment. ${ }^{35}$ Therefore, the presence of an inflammatory response confirmed an oxidative process in the hippocampus of HF-fed mice. Moreover, the inflammatory process determined might be based on the cognitive decline demonstrated by NORT test in old animals and to a higher degree in metabolically stressed C57BL/6J with respect to young ND mice. This supports that inflammation was induced by OS. RV, an antioxidant that is a well-known and 


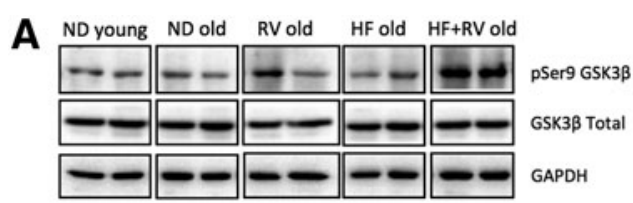

Ratio pSer9 GSK3 $\beta /$ GSK3 $\beta$ Total
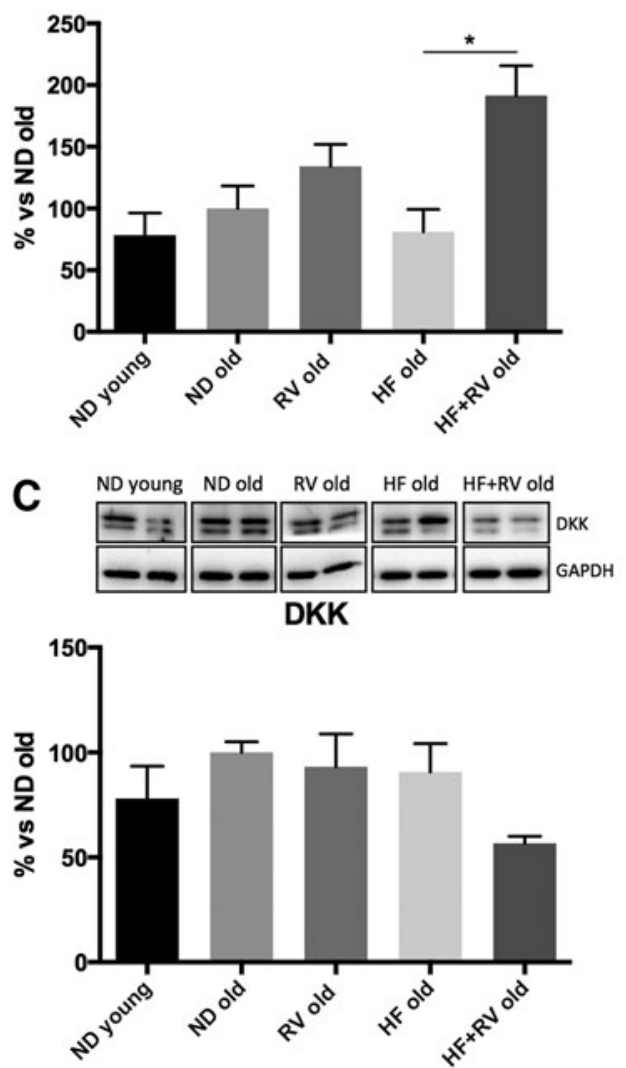

B
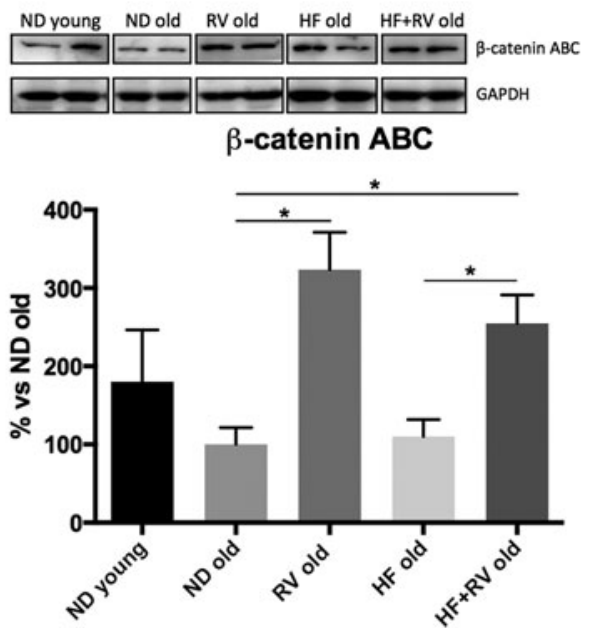

D ND young ND old RV old HF old HF+RV old

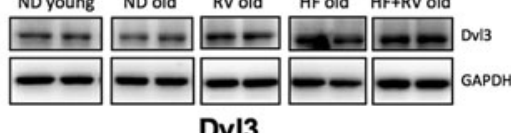

$\mathrm{Dvl3}$

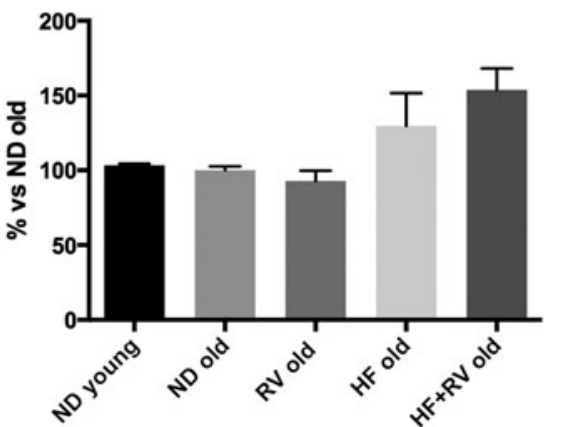

FIG. 8. Representative Western blot and quantifications for (A) Ratio p-Ser9-GSK3 $\beta / G S K 3 \beta$, (B) $\beta$-catenin ABC, (C) DKK, and (D) Dvl3 in C57BL/6J young and old fed with normal diet (ND young $n=12$ and ND old $n=9$ ), resveratrol (RV old $n=9$ ), high-fat diet (HF old $n=11$ ), or high-fat diet plus resveratrol (HF+RV old $n=9$ ). In Western blot, bars represent mean \pm SEM, and values are adjusted to $100 \%$ for levels C57BL/6J old with ND. For compared groups, we did ANOVA

AU15 One way. *Significant differences between ND old groups versus ND young and RV groups, $p<0.05$. ${ }^{\$}$ Significant differences between ND old groups versus $\mathrm{HF}$ and $\mathrm{HF}+\mathrm{RV}$ groups, $p<0.05$.

well-characterized compound, ${ }^{36}$ was able to prevent increases in the majority of the inflammation markers evaluated in this study. Therefore, the effect of RV on the inflammation process was demonstrated by a reduction in the gene expression of a number of pro-inflammatory cytokines (TNF- $\alpha, C x c l-10, I L-1, I L-6$, and $C c l 3)$.

Metabolic stress induced by $\mathrm{HF}$ has been described as affecting the mitochondrial respiratory chain. ${ }^{8,23}$ When OXPHOS chain protein elements were studied, we did not find changes in the HF compared with the ND group. However, we found changes in CII-SDHB, CIII-UQCRC2, and CIV-MTCO1 complexes in RV treatment. These results agree with those obtained in previous works ${ }^{8,37}$ and demonstrated that RV exerts consistent action on mitochondrial function.

The influence of metabolic stress and the effect of the polyphenol RV, inducing significant changes in mitochondrial function, were reinforced through Sirt1-AMPK-PGC$1 \alpha$ axis alteration. AMPK is a sensor key that controls
PGC- $1 \alpha$ activity. Results in these cellular components exhibited significant changes among young animals, old mice, and HF-fed mice. Thus, we can conclude that, although no changes in OXPHOS protein content were determined, there was mitochondrial dysfunction in aged animals. These changes were more marked in HF diet animals than in ND mice. RV was able to revert AMPK phosphorylation and achieve young animal phosphorylation levels. It was demonstrated that RV can act as a direct activator of AMPK. ${ }^{22,38}$ In our hands, apart from the antioxidant role of RV, we also demonstrated the ability of RV to act on AMPK-PGC- $1 \alpha$ axis activation and on its correlation with improvement in cognition.

SIRT1 plays a role in metabolic rate; it may be implicated in the regulation of neuronal survival ${ }^{39}$ and its overexpression in the extended life $\operatorname{span}^{40}$ induced general cognitive enhancement and protected against AD pathology in mice. ${ }^{41}$ Sirtuins are a family of nicotinamide adenine dinucleotide-dependent deacetylases that are implicated in a 

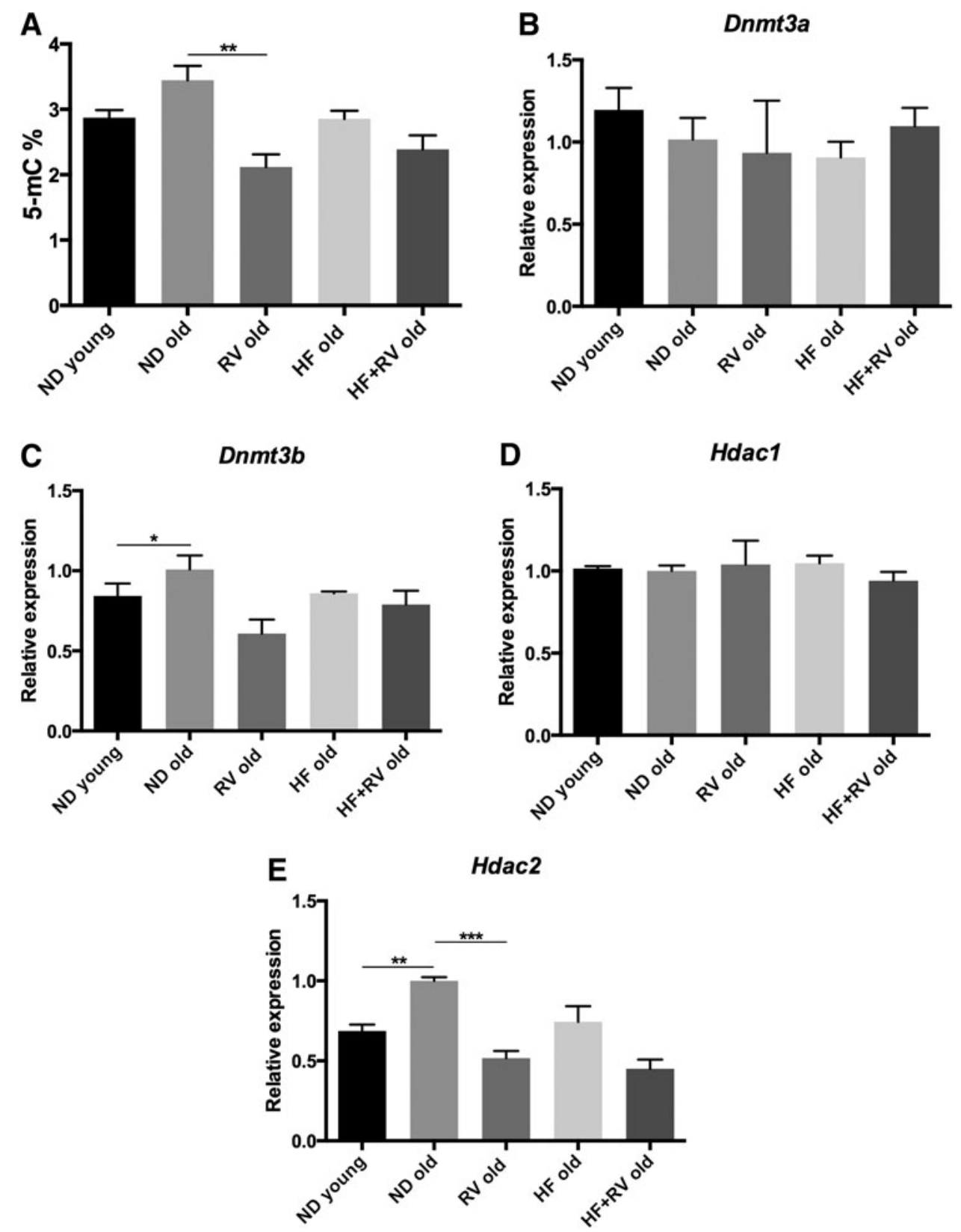

FIG. 9. (A) Global 5-methylated cytosine and gene expression for (B) Dnmt3a; (C) Dnmt3b; (D) Hdac1; and (E) Hdac2 in C57BL/6J young and old fed with normal diet (ND young $n=12$ and ND old $n=9$ ), resveratrol (RV old $n=9$ ), high-fat diet ( $\mathrm{HF}$ old $n=11$ ), or high-fat diet plus resveratrol $(\mathrm{HF}+\mathrm{RV}$ old $n=9)$. Gene expression levels were determined by real-time PCR. Mean \pm SEM performed in triplicate are represented. For compared groups, we did ANOVA One way. *Significant differences between ND old groups versus ND young and RV groups, $p<0.05, * * p<0.01, * * * p<0.001$.

AU16 \$Significant differences between ND old groups versus HF and HF+RV groups, $p<0.05$.

number of physiological and pathological processes, as the control of glucose and in lipid metabolism, cancer, genomic stability, and DNA repair. In reference to the third pillar of the metabolic sensor, Sirt1-AMPK-PGC-1 $\alpha$, we found an increase in the protein levels of SIRT1 solely in RV-fed aged animals, but not in the remainder of the experimental groups. This uniquely indicates that there were no alterations in protein expression, but does not discard changes in its activity, as will be expected by changes in AMPK phosphorylation levels, which indicate the activation levels of this kinase through LKB deacetylation. ${ }^{42,43}$ In this re- spect, RV is also described as an activator of SIRT1 and as being able to modify cellular response through AMPK activation. ${ }^{42}$

Consequently, optimal control of OS and the action on SIRT1 and AMPK tandem by RV produced a significant reduction in inflammation processes in old and in metabolically stressed old mice.

Recently, workers at the Chiaramello laboratory have launched the idea of the concept of NeuroD6-PGC-1 $\alpha$ SIRT1 as a neuroprotective axis that may be critical in coordinating mitochondrial functioning and the antioxidant 
reserve, affording cellular resilience to OS. In their work, these authors demonstrated that NeuroD6 is implicated in maintaining mitochondrial mass, intracellular ATP levels, and the expression of specific subunits of respiratory complexes under conditions of OS. ${ }^{18}$ NeuroD6 is able to maintain PGC- $1 \alpha$ and also Sirt1 expression, among other mitochondrial factors implicated in proliferation and OS. Moreover, NeuroD6 plays an intracellular ROS scavenger role in cells. In old animals, there was a diminution of NeuroD6 gene expression that RV is able to prevent, and this correlated with the effects observed for mitochondrial functioning, such as PGC- $1 \alpha$ and AMPK levels, oxidative markers (Mcp1), Wnt pathway (catenin), and Bax.

Recent studies have suggested that epigenetic mechanisms play an important role in the initiation and development of senescence. The methylation of DNA comprises one of the end points for epigenetic effectors and was commonly related with OS and inflammation processes in aging. ${ }^{10} \mathrm{It}$ was recently reported that Dnmt3b plays a role in cognitive decline in subjects with mild cognitive impairment. ${ }^{18,45}$ In this study, methylation levels in old animals were increased and normalized to young animal values by RV. In contrast, we found weak changes in DNA methylation enzymes that achieved a spurious significance among experimental groups. Histone deacetylases have been implicated in memory and cognition, and concretely, HDAC2 plays a central role in coupling lysine acetylation with synaptic plasticity. ${ }^{46}$ Results demonstrated an increase in Hdac2 gene expression in old animals compared with younger ones, and notably, RV was able to reduce this deacetylase expression in both groups of old mice. In addition to cytosolic deacetylase activity, as we previously pointed out, SIRT1 is also an enzyme participating in histone deacetylation and then in the epigenetic control of gene expression, ${ }^{47}$ and RV was able to increase Sirt1 expression in RV-fed old mice.

Therefore, epigenetic markers strongly depend on the inflammatory response, which was present in the C57BL/6J cohorts studied herein. RV was able to modify $5 \mathrm{mC}$ and Dnmt3b levels in ND old animals, indicating that an antioxidant intervention, such as $\mathrm{RV}$, produces an antiinflammatory effect and could modify epigenetic patterns as described by other authors. ${ }^{48-50}$

Because several findings suggested that OS resulted in an imbalance between DNA methylation and demethylation and histone acetylation and deacetylation associated with the activation of transcription factors when RV modifies OS may modify the expression of antioxidant enzymes, rendering a low oxidative level, and the expression of proinflammatory cytokines, controlling inflammatory processes under specific experimental conditions such as those demonstrated in the present work. Taking into account the effects on several cellular mechanisms for fighting against aging, the effect of RV on senescence and on metabolic stress during aging might be explained by a diminution in OS, which rebounds in the improvement of the inflammation process, energetic imbalance control, and finally, in epigenetic changes.

In conclusion, we demonstrated how RV induced a coordinated network of defense mechanisms against metabolic stress in the older animals, namely, it protects against old age frailty, promoting neuronal rejuvenation. Therefore, it is warranted to pursue on the study of RV brain neuroprotec- tive mechanisms to identify a feasible target to be addressed for healthy aging.

\section{Acknowledgments}

This study was supported by Spanish MINECO (SAF2016-33307) and the European Regional Development Fund. CG-F and M-P belong to 2014SGR 525 and CS to 014SGR625 from AGAUR, Catalonia. The authors thank Maggie Brunner, MD, for reviewing the language and style of the article.

\section{Author Disclosure Statement}

No competing financial interests exist.

\section{References}

1. United Nations, Department of Economic and Social Affairs, Population Division. World Population Ageing 2015 (ST/ESA/SER.A/390). 2015. Available at www.un.org/en/ development/desa/population/publications/pdf/ageing/ WPA2015_Report.pdf

2. Petersen KS, Smith C. Ageing-associated oxidative stress and inflammation are alleviated by products from grapes. Oxid Med Cell Longev 2016. [Epub ahead of print]; DOI: $10.1155 / 2016 / 6236309$.

3. De Felice FG, Lourenco MV. Brain metabolic stress and neuroinflammation at the basis of cognitive impairment in Alzheimer's disease. Front Aging Neurosci 2015;7:94.

4. Wang X, Wang W, Li L, Perry G, Lee H, Zhu X. Oxidative stress and mitochondrial dysfunction in Alzheimer' disease. Biochimica et Biophysica Acta 2014;1842:1240-1247.

5. Hagberg H, Mallard C. Effect of inflammation on central nervous system development and vulnerability. Curr Opin Neurol 2005;18:117-123.

6. Arnold SE, Lucki I, Brookshire BR, Carlson GC, Browne CA, Kazi H, Bang S, Choi BR, Chen Y, McMullen MF, Kim SF. High fat diet produces brain insulin resistance, synaptodendritic abnormalities and altered behavior in mice. Neurobiol Dis 2014;67:79-87.

7. Petrov D, Pedrós I, Artiach G, Sureda FX, Barroso E, Pallàs M, Casadesús G, Beas-Zarate C, Carro E, Ferrer I, Vazquez-Carrera M, Folch J, Camins A. High-fat dietinduced deregulation of hippocampal insulin signaling and mitochondrial homeostasis deficiencies contribute to Alzheimer disease pathology in rodents. Biochim Biophys Acta 2015;1852:1687-1699.

8. Palomera-Avalos V, Griñán-Ferré C, Puigoriol-Illamola D, Camins A, Sanfeliu C, Canudas AM, Pallàs M. Resveratrol protects SAMP8 brain under metabolic stress: Focus on mitochondrial function and Wnt pathway. Mol Neurobiol 2016. [Epub ahead of print]; DOI:10.1007/s12035-0169770-0.

9. Farias N, Ho N, Butler S, Delaney L, Morrison J, Shahrzad $\mathrm{S}$. The effects of folic acid on global DNA methylation and colonosphere formation in colon cancer cell lines. J Nutr Biochem 2015;26:818-826.

10. Vucetic Z, Kimmel J, Reyes TM. Chronic high-fat diet drives postnatal epigenetic regulation of $\mu$-opioid receptor in the brain. Neuropsychopharmacology 2011;36:1199-1206.

11. Gu X, Sun J, Li S, Wu X, Li L. Oxidative stress induces DNA demethylation and histone acetylation in SH-SY5Y cells: Potential epigenetic mechanisms in gene transcription in $\mathrm{A} \beta$ production. Neurobiol Aging 2012;34:1069-1079. 
12. Sanchez-Mut JV, Graff J. Epigenetic Alterations in Alzheimer's Disease. Front Behav Neurosci 2015;9:347.

13. Richardson B. Impact of aging on DNA methylation. Aging Res Rev 2003;2:245-261.

14. Bruel-Jungerman E, Davis S, Rampon C, Laroche S. Longterm potentiation enhances neurogenesis in the adult dentate gyrus. J Neurosci 2006;26:5888-5893.

15. Gould E, Beylin A, Tanapat P, Reeves A, Shors TJ. Learning enhances adult neurogenesis in the hippocampal formation. Nat Neurosci 1999;2:260-265.

16. Kempermann G, Chesler EJ, Lu L, Williams RW, Gage FH. Natural variation and genetic covariance in adult hippocampal neurogenesis. Proc Natl Acad Sci 2006;103:780_ 785.

17. van Praag H, Shubert T, Zhao C, Gage FH. Exercise enhances learning and hippocampal neurogenesis in aged mice. J Neurosci 2005;25:8680-8685.

18. Uittenbogaard U, Baxter KK, Chiaramello A. The neurogenic basic helix-loop-helix transcription factor NeuroD6 confers tolerance to oxidative stress by triggering an antioxidant response and sustaining the mitochondrial biomass. ASN Neuro 2010;2:e0034.

19. Lagouge M, Argmann C, Gerhart-Hines Z, Meziane H, Lerin C, Daussin F, Messadeq N, Milne J, Lambert P. Resveratrol improves mitochondrial function and protects against metabolic disease by activating SIRT1 and PGC- $1 \alpha$. Cell 2006;127:1109-1122.

20. Pallàs M, Porquet D, Vicente A, Sanfeliu C. Resveratrol: New avenues for a natural compound in neuroprotection. Curr Pharm Des 2013;19: 6726-6731.

21. Porquet D, Griñán-Ferré C, Ferrer I, Camins A, Sanfeliu C, Del Valle J, Pallàs M. Neuroprotective role of transresveratrol in a murine model of familial Alzheimer's disease. J Alzheimer's Dis 2014;42:1209-1220.

22. Fullerton MD, Steinberg GR. SIRT1 takes a backseat to AMPK in the regulation of insulin sensitivity by resveratrol. Diabetes 2010;59:551-553.

23. Jeon BT, Jeong EA, Shin HJ, Lee Y, Lee DH, Kim HJ, Kang SS, Cho GJ, Choi WS, Roh GS. Resveratrol attenuates obesity-associated peripheral and central inflammation and improves memory deficit in mice fed a high-fat diet. Diabetes 2012;61:1444-1454

24. Ayala JE, Samuel VT, Morton GJ, Obici S, Croniger CM, Shulman GI, Wasserman DH, McGuinness OP; NIH Mouse Metabolic Phenotyping Center Consortium. Standard operating procedures for describing and performing metabolic tests of glucose homeostasis in mice. Dis Model Mech 2010;3:525-534.

25. Ennaceur A, Delacour J. A new one-trial test for neurobiological studies of memory in rats. Behavioral data. Behav Brain Res 1988;31:47-59.

26. Vorhees CV, Williams MT. Morris water maze: Procedures for assessing spatial and related forms of learning and memory. Nat Protoc 2006;1:848-858.

27. Hall CS. Emotional behavior in the rat. I. Defecation and urination as measures of individual differences in emotionality. J Comp Psychol 1934;8:385-403.

28. Fjell AM, McEvoy L, Holland D, Dale AM, Walhovd KB, for the Alzheimer's Disease Neuroimaging Initiative. What is normal in normal aging? Effects of aging, amyloid and Alzheimer's disease on the cerebral cortex and the hippocampus. Prog Neurobiol 2014;117:20-40.

29. Fischer R, Maier O. Interrelation of oxidative stress and inflammation in neurodegenerative disease: Role of TNF.
Oxid Med Cell Long 2015. [Epub ahead of print]; DOI: 10.1155/2015/610813.

30. Godbout JP, Chen J, Abraham J, Richwine AF, Berg BM, Kelley KW, Johnson RW. Exaggerated neuroinflammation and sicknes behavior in aged mice following activation of the peripheral innate immune system. FASEB J 2005;19: 1329-1331.

31. Prior M, Chiruta C, Currais A, Goldberg J, Ramsey J, Dargusch R, Maher PA, Schubert D. Back to the future with phenotypic screening. ACS Chem Neurosci 2014;5: 503-513.

32. Currais A, Goldberg J, Farrokhi C, Chang M, Prior M, Dargusch R, Daugherty D, Armando A, Quehenberger O, Maher P, Schubert D. A comprehensive multiomics approach toward understanding the relationship between aging and dementia. Aging 2015;7:937-955.

33. Ohta S, Ohsawa I, Kamino K, Ando F, Shimokata H. Mitochondrial ALDH2 deficiency as an oxidative stress. Ann N Y Acad Sci 2004;1011:36-44.

34. Chen CH, Batista-Ferreira JB, Gross ER, Mochly-Rosen D. Targeting aldehyde dehydrogenase 2: New therapeutic opportunities. Physiol Rev 2014;94:1-34.

35. Kriz J. Inflammation in ischemic brain injury: Timing is important. Crit Rev Neurobiol 2006;18:145-157.

36. Tung BT, Rodríguez-Bies E, Talero E, Gamero-Estévez E, Motilva V, Navas P, López-Lluch G. Anti-inflammatory effect of resveratrol in old mice liver. Exp Gerontol 2015; 64:1-7.

37. De Oliveira MR, Nabavi SF, Manayi A, Daglia M, Hajheydari Z, Nabavi SM. Resveratrol and the mitochondria: From triggering the intrinsic apoptotic pathway to inducing mitochondrial biogenesis, a mechanistic view. Biochimica et Biophysica Acta 2016;1860:727-745.

38. Baur JA, Pearson KJ, Price NL, Jamieson HA, Lerin C, Kalra A, Prabhu VV, Allard JS, Lopez-Lluch G, Lewis K, Pistell PJ, Poosala S, Becker KG, Boss O, Gwinn D, Wang M, Ramaswamy S, Fishbein KW, Spencer RG, Lakatta EG, Le Couter D, Shaw RJ, Navas P, Puigserver P, Ingram DK, de Cabo R, Sinclair DA. Resveratrol improves health and survival of mice on a high-caloric diet. Nature 2006; 444:16.

39. Cardinale A, de Stefano MC, Mollinari C, Racaniello M, Garaci E, Merlo D. Biochemical characterization of sirtuin 6 in the brain and its involvement in oxidative stress response. Neurochem Res 2015;40:59-69.

40. Duan W. Sirtuins: From metabolic regulation to brain aging. Front Aging Neurosci 2013;5:36.

41. Corpas R, Revilla S, Ursulet S, Castro-Freire M, Kaliman \AU8 P, Petegnief V, Giménez-Llort L, Sarkis C, Pallàs M, Sanfeliu C. SIRT1 overexpression in mouse hippocampus induces cognitive enhancement through proteostatic and neurotrophic mechanisms. Mol Neurobiol 2016.

42. Ruderman NB, Xu XJ, Nelson L, Cacicedo JM, Saha AK, Lan F, Ido Y. AMPK and SIRT1: A long-standing partnership? Am J Physiol Endocrinol Metab 2010;298:E751E760.

43. Price NL, Gomes AP, Ling AJ, Duarte FV, MartinMontalvo A, North BJ, Agarwal B, Ye L, Ramadori G, Teodoro JS, Hubbard BP, Varela AT, Davis JG, Varamini B, Hafner A, Moaddel R, Rolo AP, Coppari R, Palmeira CM, de Cabo R, Baur JA, Sinclair DA. SIRT1 is required for AMPK activation and the beneficial effects of resveratrol on mitochondrial function. Cell Metab 2012;15: 675-690. 
AU9 44. Chouliaras L, Kenis G, Visser PJ, Scheltens P, Tsolaki M, Jones RW, Kehoe PG, Graff C, Girtler NG, Wallin ÅK, Rikkert MO, Spiru L, Elias-Sonnenschein LS, Ramakers IH, Pishva E, van Os J, Steinbusch HW, Verhey FR, van den Hove DL, Rutten BP. DNMT3A moderates cognitive decline in subjects with mild cognitive impairment: Replicated evidence from two mild cognitive impairment cohorts. Epigenomics 2015;7:533-537.

45. Córdova-Palomera A, Fatjó-Vilas M, Kebir O, Gastó C, Krebs MO, Fañanás L. Polymorphic variation in the epigenetic gene DNMT3B modulates the environmental impact on cognitive ability: A twin study. Eur Psychiatry 2015; 30:303-308.

46. Penney J, Tsai LH. Histone deacetylases in memory and cognition. Sci Signal 2014;7:re12.

47. Heyward FD, Gilliam D, Coleman MA, Gavin CF, Wang J, Kaas G, Trieu R, Lewis XJ, Moulden J, Sweatt JD. Obesity weighs down memory through a mechanism involving the neuroepigenetic dysregulation of Sirt1. J Neurosci 2016;36: 1324-1335.

48. Junien C. Impact of diets and nutrients/drugs on early epigenetic programming. J Inherit Metab Dis 2006;29: 359-365.
49. Farghali H, Canová NK, Lekic N. Resveratrol and related compounds as antioxidants with an allosteric mechanism of action in epigenetic drug targets. Physiol Res 2013;62: $1-13$.

50. Sezgin Z, Dincer Y. Alzheimer's disease and epigenetic diet. Neurochem Int 2014;78:105-116.

Address correspondence to: Mercè Pallàs \AU10 Pharmacology Section Department of Pharmacology, Toxicology and Therapeutic Chemistry Institute of Neuroscience University of Barcelona Avinguda Joan XXIII $s / n$ Barcelona 08028

Spain

E-mail: pallas@ub.edu

Received: September 22, 2016 Accepted: November 21, 2016 


\section{AUTHOR QUERY FOR REJ-2016-1885-VER9-PALOMERA-AVALOS_1P}

AU1: Please note that gene symbols in any article should be formatted as per the gene nomenclature. Thus, please make sure that gene symbols, if any in this article, are italicized.

AU2: Please review all authors' surnames for accurate indexing citations.

AU3: Please confirm the correctness of authors' affiliations.

AU4: Please fix the expansion of the acronym "ROS": "radical oxygen species" or "reactive oxidative species"?

AU5: Please define NE, SE, SW, and NW.

AU6: Please fix the expansion of the acronym "RT-PCR": "reverse transcription-polymerase chain reaction" or "Realtime polymerase chain reaction"?

AU7: Disclosure statement accurate? If not, please amend as needed.

AU8: In Ref. 41, please mention the volume number and page range. If these are unavailable, please supply the article's full DOI number.

AU9: Ref. 44 has been deleted as it was a duplicate of Ref. 18, and the Refs. 45-51 (“45-44,” “46-45," “47-46,” " 4847," “49-48," “50-49," and "51-50") citations in the text have been renumbered to maintain sequential order. Please check. Also, please cite Ref. 44 in the text.

AU10: Please confirm the address of correspondence.

AU11: Please note that "Tables 1A, 1B, and 2" have been renumbered as "Tables 1, 2, and 3," respectively. Please check.

AU12: Please expand QRT-PCR.

AU13: Please expand QRT-PCR.

AU14: Asterisk and Dollar are described in the legend of Figure 1, but not given in the artwork of the same. Please check. AU15: Dollar is described in the legend of Figure 8, but not given in the artwork of the same. Please check.

AU16: Dollar is described in the legend of Figure 9, but not given in the artwork of the same. Please check. 